\title{
Synchrony with shunting inhibition
}

\section{Sachin S Talathi*, Dong-Uk Hwang, Abraham Miliotis, Paul R Carney and William L Ditto}

\author{
Address: Department of Biomedical Engineering, University of Florida, Gainesville, Florida, 32611, USA \\ Email: Sachin S Talathi* - sachin.talathi@bme.ufl.edu \\ * Corresponding author
}

from Eighteenth Annual Computational Neuroscience Meeting: CNS*2009

Berlin, Germany. 18-23 July 2009

Published: 13 July 2009

BMC Neuroscience 2009, 10(SuppI I):P233 doi:I0.II86/I47I-2202-I0-SI-P233

This abstract is available from: http://www.biomedcentral.com/I47I-2202/I0/SI/P233

(C) 2009 Talathi et al; licensee BioMed Central Ltd.

Spike time response curves (STRC's) are used to study the influence of synaptic stimuli on the firing times of a neuron oscillator without the assumption of weak coupling. They allow us to approximate the dynamics of synchronous state in networks of neurons through a discrete map. Linearization about the fixed point of the discrete map can then be used to predict the stability of patterns of synchrony in the network. General theory for taking into account the contribution from higher order STRC terms, in the approximation of the discrete map for coupled neuronal oscillators in synchrony is still lacking. Here we present a general framework to account for higher order STRC corrections in the approximation of discrete map to determine the domain of 1:1 phase locking state in the network of two interacting neurons. We begin by demonstrating that the effects of synaptic stimuli through a shunting synapse to a neuron firing in the gamma frequency band $(20-80 \mathrm{~Hz})$ last for three consecutive firing cycles (See Figure 1). We then show that the discrete map derived by taking into account the higher order STRC contributions is successfully able predict the domain of synchronous 1:1 phase locked state in a network of two heterogeneous interneurons coupled through a shunting synapse (See Figure 2).

\section{Acknowledgements}

The work was funded through the grant from the Office of Naval Nesearch (N000I4-02-I-I0I9).

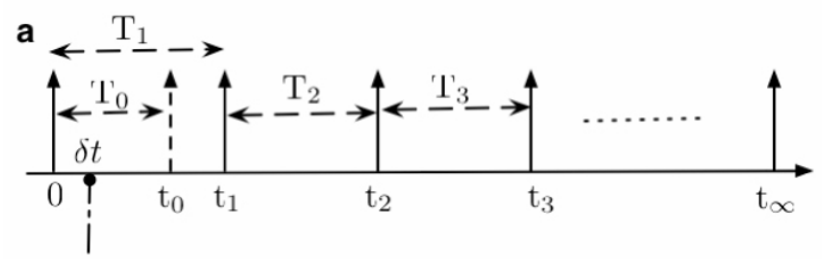

b

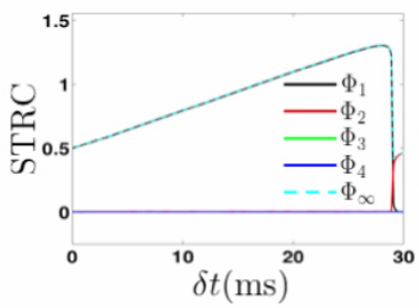

c

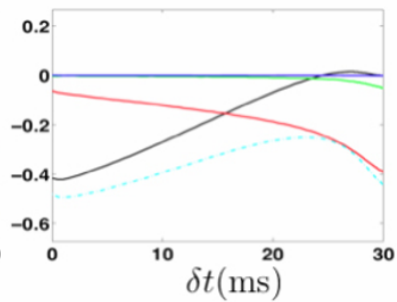

Figure I

(a) Schematic diagram demonstrating the effect of perturbation received by a spiking neuron at time $t$. The cycle containing the perturbation defines the first order STRC and the subsequent cycles define the higher order STRC terms. (b) The STRC's computed for neuron receiving perturbation through a GABA mediated synapse at hyperpolarizing reversal potential of $E_{R}=-80 \mathrm{mV}$. (c) The STRC's computed for neuron receiving perturbation through a $G A B A_{A}$ mediated synapse at shunting reversal potential of $E_{R}=-55 \mathrm{mV}$. The resting membrane potential of neuron is $-65 \mathrm{mV}$. The synaptic parameters are $t_{R}=0.1 \mathrm{~ms}, t_{D}=8 \mathrm{~ms}, g_{s}=0.15 \mathrm{mS} /$ $\mathrm{cm}^{2}$. The intrinsic period of firing for the neuron was $T_{0}=3 \mathrm{I}$ ms. 

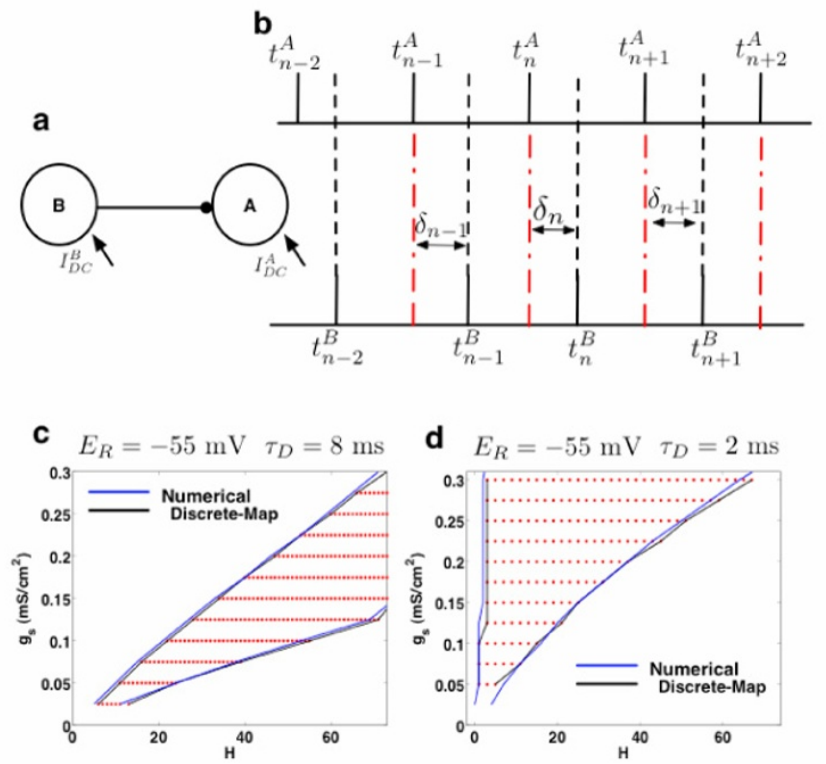

\section{Figure 2}

(a) Schematic diagram of the network considered: Two neurons with heterogeneity in their firing rates coupled through a shunting inhibitory synapse. (b) Schematic diagram representing spike timing for neurons $A$ and $B$ when they are phase locked in I:I synchrony. In (c) and (d) we show domain of I:I synchrony (phase locked state) estimated through STRC's from the discrete map derived by taking into account higher order STRC contributions for shunting synapse. 